\title{
A Comparison of Conventional Screen-Film Radiography and Hard Copy of Computed Radiography in Full and Two-Thirds Sizes in Detection of Interstitial Lung Disease
}

\author{
Hiroshi Kondoh, Junpei Ikezoe, Kiyonari Inamura, Chikazumi Kuroda, and Takahiro Kozuka
}

\begin{abstract}
This study examined whether hard-copy radiographs produced from computed radiography (CR) images show the subtle interstitial pulmonary disease equally well to conventional screen-film radiographs, because a digital radiography should be chosen for introduction of the digital picture archiving and communication system (PACS) for the new Osaka University Hospital. ${ }^{1,2}$ Eleven radiologists examined 20 abnormal and 20 control chest radiographs presented in each of three groups: conventional screen-film radiographs and two sizes of hard-copy radiographs made from CR images. This study of digital image quality of chest examinations found that some findings on conventional screen-film radiography images are not reproduced by current CR $(2,000 \times 2,000 \times 10$ bits in matrix), especially when the experienced radiologists were observed. This finding suggested improvements are needed in CR before CR of chest should fully replace conventional screen-film radiography.

Copyright $\odot 1994$ by W.B. Saunders Company
\end{abstract}

KEY WORDS: Screen film radiography, computed radiography (CR), storage phosphor system, observer performance test, receiver operating characteristic (ROC) study, interstitial lung disease.

$\mathbf{I}$ $\mathrm{N}$ THIS STUDY, the diagnostic accuracy of photostimulable phosphor images printed on hard copy with a $2,000-\times 2,000$-pixel matrix and 10-bit system (Fuji Computed Radiography (CR), Fuji Film Industry Co, Ltd, Tokyo, Japan) ${ }^{1,2}$ was compared with conventional screenfilm chest radiographs. Detection of subtle interstitial pulmonary abnormalities was studied to evaluate differences among radiologists in interpreting conventional radiographs against two sizes of storage phosphor (CR) images on film.

\section{MATERIALS AND METHODS}

The study set comprises three groups of images: (1) conventional film radiographs $(14 \times 17$ inches, conventional); (2) hard copy images $(14 \times 17$ inches, $0.2 \mathrm{~mm}$ pixel size, $C R \times 1)$; and (3) minified images $(9 \times 11$ inches, 0.1 $\mathrm{mm}$ pixel size with interpolation from same digital data as $\mathrm{CR} \times 1$, about $2 / 3$ of the size of images on film, $\mathrm{CR} \times 2 / 3$ ). Subjectively optimized image processing was done under manual control for each image in both groups.

The study images were of 20 patients with subtle interstitial pulmonary lesions and 20 control patients without pulmonary lesions. The various kinds of interstitial pulmonary diseases were included. These were 8 collagen diseases, 5 hypersensitivity pneumonitis or diffuse panbronchi- olitis, and 1 each of pulmonary metastatic calcification, sarcoidosis, alveolar proteinosis, eosinophilic granuloma, and the remaining 2 interstitial diseases of unknown cause. Some of the radiographs had fine reticular infiltrates, ill-defined faint small nodules up to $3 \mathrm{~mm}$ in diameter, and/or groundglass opacities. All 40 patients underwent computed tomography (CT) of the chest, and CT images were used as the gold standard for this study.

All conventional and storage phosphor images were obtained with $130 \mathrm{KVp}, 1.0-\mathrm{mm}$ nominal focus size, $200-\mathrm{cm}$ film-focus distance, and a 12:1 Bucky. CR images were obtained by storage phosphor imaging plates and were digitized into $0.2-\mathrm{mm} \times 10$-bit pixel matrix and printed on film.

Observations were performed by 11 radiologists. Seven were experienced radiologists, and the other 4 were junior staff or residents. They were informed about the kinds of abnormalities included in the study and that the changes were subtle. Each was asked to describe the presence of abnormalities to a five-level scale of confidence. Observer performance for the three groups of images was tested according to receiver operating characteristic (ROC) analysis. Perceptual accuracy was described by the area under the ROC curve. Az values (averaged areas under the ROC curve) were used to test the significance of differences in performance by means of paired $t$-test.

\section{RESULTS}

The observations from the 40 cases in each of the three groups by the 11 radiologists were analyzed by the ROC method. Figure 1 shows the ROC curves for the three groups of images studied. The measured areas under the ROC curve for each of the 11 radiologists are shown in Table 1 . There was no significant difference between the two CR groups, $\mathrm{CR} \times 1$ and $\mathrm{CR} \times$ $2 / 3$. There also was no significant difference between the minified CR $\times 2 / 3$ group and the screen-film radiographs (conventional). There was a significant difference between the screen-

From the Department of Radiology, Faculty of Medicine; and the Department of Radiological Technology and Biomedical Engineering, School of Allied Health Sciences, Faculty of Medicine, Osaka University, Osaka, Japan

Address reprint requests to Hiroshi Kondoh, MD, Department of Radiology, Osaka University Medical School, 2-2, Yamada-oka, Suita, Osaka 565, Japan.

Copyright 1994 by W.B. Saunders Company

0897-1889/94/0704-0001\$3.00/0 


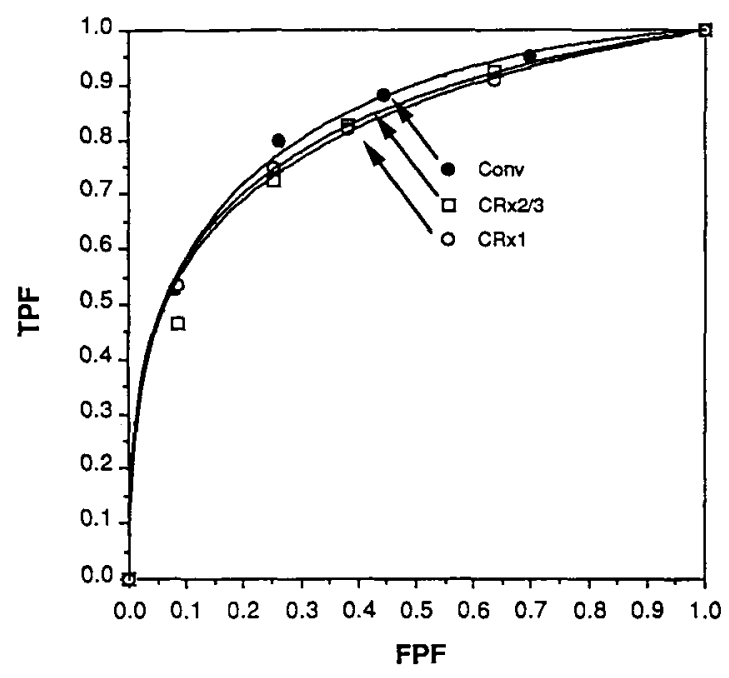

Fig 1. Diagnostic accuracy for diffuse lung disease by 11 radiologists. TPF, true position fraction; FPF, false positive fraction.

film radiographs (conventional) and the fullsize hard-copy images $(\mathrm{CR} \times 1)$.

Figure 2 shows the pooled ROC curves for the seven experienced radiologists. For this group, the differences between the screen-film radiographs (conventional) and both $\mathrm{CR}$ hardcopy groups, $\mathrm{CR} \times 1$ and $\mathrm{CR} \times 2 / 3$, were significant at .05 level (Table 2). On the other hand, there was no significant difference in observer performance of the residents between all three groups (Table 3 ).

\section{DISCUSSION}

The storage phosphor system has wide dynamic range, but the modulation transfer func-

\begin{tabular}{|cccc}
$\begin{array}{r}\text { Table 1. Areas Under RoC Curve for 11 Radiologists } \\
\text { According to Image Modalities }\end{array}$ \\
\hline Reader & Conventional & CR $\times 1$ & CR $\times 2 / 3$ \\
\hline 1 & .918 & .899 & .852 \\
2 & .882 & .890 & .871 \\
3 & .876 & .858 & .868 \\
4 & .904 & .843 & .892 \\
5 & .894 & .801 & .795 \\
6 & .892 & .851 & .857 \\
7 & .880 & .905 & .865 \\
8 & .861 & .774 & .771 \\
9 & .831 & .815 & .813 \\
10 & .787 & .757 & .769 \\
11 & .749 & .736 & .812 \\
Mean \pm SEM & $.861 \pm .016$ & $.830 \pm .013$ & $.833 \pm .018$ \\
\hline
\end{tabular}

Abbreviation: NS, not significant.

* $P$ values represent mean $\pm S E M$ of each modality compared with conventional.

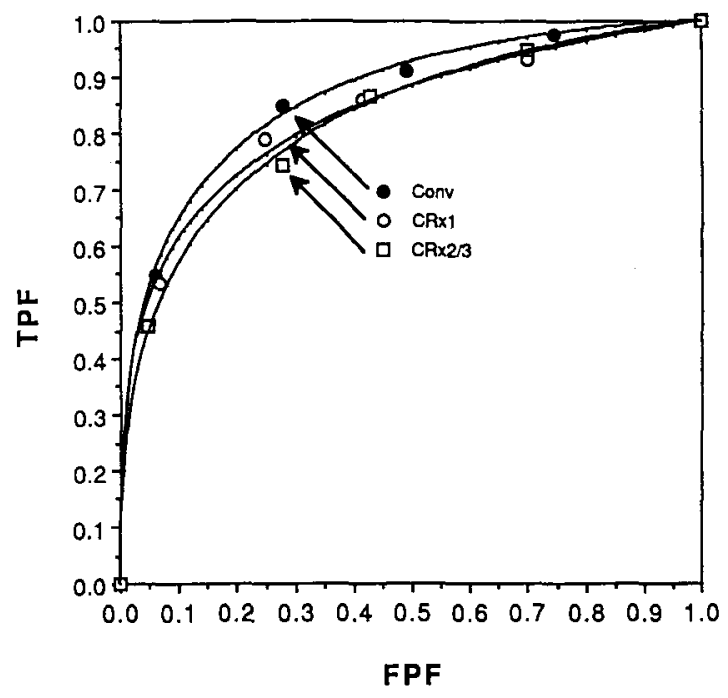

Fig 2. Diagnostic accuracy for diffuse lung disease by 7 experienced radiologists.

tion (MTF) at high frequency was reported to be lower than conventional screen-film system by Sanada et $\mathrm{al}^{3}$ and MacMahon et $\mathrm{al}^{4}$ who reported that the diagnostic accuracy of interstitial infiltrates and subtle pneumothoraces with $0.1-\mathrm{mm}$ pixel images made by film digitization was inferior to that of original conventional screen-film radiographs. On the other hand, the existence of subtle interstitial lung disease should be detected as small and vague granular or reticular shadows or haziness of vascular structure. These changes are considered to be related to the high-frequency part of MTF, compared with mass shadow, which is considered to be related to lower frequency of MTF.

We evaluated two sizes of hard copy of Fuji Computed Radiography that contained the same image data because it seemed harder to detect higher-frequency signals in the smaller image. However, our results did not indicate that. We were able to take closer position at observation in our study and the effect mentioned above was negated.

In our hospital, early detection of acute

Table 2. Area Under ROC Curve for 7 Experienced Radiologists According to Image Modalities

\begin{tabular}{lll}
\hline & Mean \pm SEM & \\
\hline CR $\times 1$ & $.847 \pm .014$ & $P=.038<.05$ \\
Conventional & $.889 \pm .005$ & $P=.019<.05$ \\
$C R \times 2 / 3$ & $.843 \pm .016$ & \\
\hline
\end{tabular}


Table 3. Area Under ROC Curve for 4 Resident Radiologists According to Image Modalities

\begin{tabular}{lll} 
& Mean \pm SEM & \\
\hline CR $\times 1$ & $.800 \pm .034$ & $P=.203: \mathrm{NS}$ \\
Conventional & $.812 \pm .029$ & $P=.852: \mathrm{NS}$ \\
$\mathrm{CR} \times 2 / 3$ & $.816 \pm .021$ & \\
\hline
\end{tabular}

Abbreviation: NS, not significant.

subtle interstitial lung abnormalities is important because we have many patients with collagen disease who have a possibility of acute progression of pulmonary involvements, and immunocompromized patients who are treated with chemotherapy for malignancies and who might contract opportunistic infections and hypersensitivity pneumonitis. So the detection of subtle interstitial lung disease seemed not only to be one of the weak points of the CR system, but also to be the important disease.

In our results, the area under ROC for four residents did not show a significant difference between the conventional system and CR images at a lower level of accuracy. On the other hand, the area under ROC for experienced radiologists showed the conventional system was superior to CR images at a higher level. Our results indicated that the detection or demanded image quality were dependent on the level of observers' performance.

Therefore, we concluded we were not fully satisfied with the present $\mathrm{CR}$ system (2,000 $\times 2,000$-pixel, 10 bits $)$. Consequently, we needed a higher-quality digital radiography system to completely replace the conventional screen-film system.

In fact, we adopted the new high-resolution storage phosphor system (Fuji Film Industry Co Ltd) in the new hospital, which had 4,000- $\times$ 5,000-pixel matrix and 10 bits in the depth to improve image quality in cooperation with Fuji Film Industry Co Ltd. The data of an image is about 40 Mbyte and must be compressed for transmission and storage to PACS. We must use the hard copy for primary diagnosis and the PACS is then used for distribution of reference images.

\section{REFERENCES}

1. Kuroda C, Kondoh H, Takeda H, et al: Planning for PACS at Osaka University Hospital. Comput Methods Programs Biomed 36:147-150, 1991

2. Inamura K: PACS in Japan. Proc Comput Assist Radiol 91:383-390, 1991

3. Sanada S, Doi K, Xu X, et al: Comparison of imaging properties of a computed radiography system and screenfilm systems. Med Phys 18:414-420, 1991

4. MacMahon H, Vyborny CJ, Metz CE, et al: Digital radiography of subtle pulmonary abnormalities: An ROC study of the effect of pixel size on observer performance. Radiology 158:27-33, 1986 